\title{
Doubling effort makes up for 6 IQ points
}

\author{
M.V. Simkin and V.P. Roychowdhury \\ Department of Electrical and Computer Engineering, University of California, Los Angeles, CA \\ 90095-1594
}

We report the results of experiments where we compared with the baseline test subjects' IQs measured in two non-standard ways: by doubling the allotted time and by doubling the manpower. As a baseline, we administered the test the regular way, where we allowed each subject the standard time prescribed in test description to complete the test. We then performed two different experiments. First, we paired the subjects of roughly equal IQ and then asked them to do the test in collaboration within the prescribed time. In the second experiment the subjects did the test solitary but were allowed twice the prescribed time. In both cases, when we doubled the allotted time, or when we doubled the manpower, we got on average a 6 point $(0.4$ Standard Deviation) increase in measured IQ.

What is an IQ? Everyone has heard of it. Some also know that its distribution is a bell curve (Herrnstein and Murray, 1994). A lesser number of people know where the curve does come from. It comes from the measurement of intelligence in children (Eysenck and Fulker, 1979). If a child of 5 scores on an intelligence test as an average child of 6 he is said to have a mental age of 6 . One defines IQ as a ratio of mental age to chronological age multiplied by 100. In given example it is 120 . Experiments show that children's IQs follows approximately Gaussian distribution with the standard deviation of about 15 (Eysenck and Fulker, 1979). After a certain age the test scores stop growing and for the adults this definition of IQ is meaningless. To overcome this hardship the testers switch to percentile ranks ${ }^{1}$. About $9 \%$ of 5-year-olds score a mental age 6 or above. Or in other words have an IQ of 120 or more. So, if an adult scores in top $9 \%$ of the adults he is said to have an IQ of 120. If he had kept his percentile rank among his age peers throughout all his life ${ }^{2}$, he should have had a mental age of 6 at the chronological age 5 .

This is pretty much all we fundamentally know about IQ. Researchers get into debates on the role of genetic and environmental factors that contribute to it, and its role in the class structure in American life (Herrnstein and Murray, 1994; Gould, 1996; Jensen, 1998). However, we don't know much about IQ beyond mere percentile rankings. There is not a lot of information in them. We measure engine power in horse powers, not as a percentile rank among all engines. We measure computer power in MIPS (Million Instructions Per Second) or FLOPS (Floating Point Operations Per Second) not as a percentile rank among other computers. Can we say anything more about the intellectual power beyond mere percentile ranking? This study attempts to do this in an experiment where we look how the measured IQ increase when we allow two people (i.e. doubling manpower) to collaborate on

\footnotetext{
${ }^{1}$ In practice they do it in a more cumbersome way. They adjust the test questions so that the resulting raw score distribution becomes roughly Gaussian and afterward rescale the raw scores so that the standard deviation becomes 15 (Eysenck and Fulker, 1979) .

${ }^{2}$ This rarely happens since the correlation between the adult IQ and the IQ at the age of 5 is only about 0.5 (Mackintosh, 1998).
} 
the test. In another experiment we check how the measured IQ increases when we double the time allowance for the test.

\section{Experiment 1}

All the test subjects in this and other experiments were UCLA graduate students who volunteered to take part in the experiment for $\$ 30$. In the beginning the 10 subjects received example test questions with answers (the Examples section of Eysenck and Evans, 1994) which they could study for about 15 minutes. Afterward they did \#1 test from Eysenck and Evans (1994). Next the subjects formed 5 pairs and collaboratively did tests \#2 and \#3. Finally, the subjects separately did test \#4. The standard prescribed by the test book (Eysenck and Evans 1994) of 30-minute allowance was used each time. There were rest breaks after each test so the whole procedure took 3 hours out of which two hours were used for testing. The results are in Table 1. The meaning of the numbers in columns 1, 2, 3, 4 is self-evident. The value in the last column, $\Delta$, we computed as (taking as an example Subjects 1 and 2):

$$
\Delta=\frac{S^{2}+S^{3}}{2}-\frac{S_{1}^{1}+S_{2}^{1}+S_{1}^{4}+S_{2}^{4}}{4} .
$$

Here $S^{2}$ and $S^{3}$ are the scores of the pair of the subjects 1 and 2 on tests 2 and 3. $S_{1}^{1}$ and $S_{2}^{1}$ are the individual scores of subjects 1 and 2 on test 1 . Similarly, $S_{1}^{4}$ and $S_{2}^{4}$ are the scores on test 4 . The mean increase in effective IQ due to collaboration is 7.3 points. The bottom row "average" lists the arithmetic means of the numbers in each column. The standard error of the average $\Delta$ is 1.9 points.

Table 1. The clear columns show the IQ scores received by the subjects working on the tests individually. The shaded columns show the scores received by the subjects collaborating in pairs. On average the effective IQ of a pair is 7.3 points higher than the average individual IQ of the members of the pair.

\begin{tabular}{|c|c|c|c|c|c|}
\hline Subject \# & 1 & 2 & 3 & 4 & $\Delta$ \\
\hline 1 & 120 & \multirow{2}{*}{128.3} & \multirow{2}{*}{131.7} & 133.3 & \multirow{2}{*}{6.3} \\
\hline 2 & 113.3 & & & 128.3 & \\
\hline & & \multirow{3}{*}{123.3} & \multirow{3}{*}{125.0} & & \multirow{3}{*}{2.5} \\
\hline 3 & 115 & & & 125.0 & \\
\hline 4 & 116.7 & & & 130.0 & \\
\hline 5 & 116.7 & \multirow{2}{*}{133.3} & \multirow{2}{*}{128.3} & 128.3 & \multirow{2}{*}{7.5} \\
\hline 6 & 120.0 & & & 128.3 & \\
\hline 7 & 116.7 & \multirow{2}{*}{128.3} & \multirow[b]{2}{*}{130.0} & 128.3 & \multirow[b]{2}{*}{6.3} \\
\hline 8 & 116.7 & & & 130.0 & \\
\hline & & \multirow{3}{*}{140.0} & \multirow{3}{*}{131.7} & & \multirow{3}{*}{14.2} \\
\hline 9 & 111.7 & & & 131.7 & \\
\hline 10 & 115.0 & & & 128.3 & \\
\hline average & 116.2 & 130.7 & 129.3 & 129.2 & 7.3 \\
\hline
\end{tabular}

One may argue that it is more reasonable to compare the effective IQ of the pair not with the average IQs of its members, but with the highest individual IQ in the pair. This we can compute as 


$$
\Delta^{\prime}=\frac{S^{2}+S^{3}}{2}-\max \left(\frac{S_{1}^{1}+S_{1}^{4}}{2} ; \frac{S_{2}^{1}+S_{2}^{4}}{2}\right)
$$

The average $\Delta^{\prime}$ is 6.2 points, not much different from the average $\Delta$. Apparently because all the subjects had close IQs.

Another issue is that the average score of 10 subjects on test 4 is 13 points above the average score on test 1 . The reason for it is unclear. It could be that test 4 is just easier than test 1 . It could also be that that the score increases with practice. Albeit we do not see any increase from tests 2 to 3 . It could also be that the subjects are "warming-up" during the first test and perform the rest of them with their full intellectual power.

\section{Experiment 2}

Some of the subjects took part in Experiment 1, so we used new tests. The 8 subjects did \#5 test from Eysenck and Evans (1994) using 30 minutes, as prescribed by the book. Afterward they did tests \#6 and \#7 taking 1 hour (twice the prescribed time) for each of them. Finally, the subjects did test \#8 in 30 minutes. The results are in Table 2 . The $\Delta$ values in the last column we computed as

$$
\Delta=\frac{S^{6}+S^{7}}{2}-\frac{S^{5}+S^{8}}{2}
$$

where $S^{n}$ are each subject's scores on the $n^{\text {th }}$ test. The average increase in effective IQ due to testing time increase is 7.2 points, the standard error is 1.1 . Note, however, that just like before we see the increase in the average test score between the first and the last tests of the day by 12.1 points. Both tests the subjects completed during the standard time allowance, nonetheless the difference in average scores exceeds $\Delta$, the gain due to time doubling.

Table 2. The clear columns show the IQ scores received by the subjects completing the test during standard time. The shaded columns show the scores received when the time allowance was increased twice. On average, the effective IQ measuured when the test time was increased twice is 7.2 points higher than the average IQ measured normally.

\begin{tabular}{|c|c|c|c|c|c|}
\hline $\begin{array}{c}\text { Test \# } \\
\text { Subject \# }\end{array}$ & 5 & 6 & 7 & 8 & $\Delta$ \\
\hline 1 & 120.0 & 126.7 & 121.7 & 116.7 & 5.8 \\
\hline 2 & 118.3 & 131.7 & 133.3 & 128.3 & 9.2 \\
\hline 3 & 118.3 & 130.0 & 130.0 & 130.0 & 5.8 \\
\hline 4 & 106.7 & 121.7 & 123.3 & 123.3 & 7.5 \\
\hline 5 & 120.0 & 131.7 & 131.7 & 133.3 & 5.0 \\
\hline 6 & 126.7 & 135.0 & 136.7 & 138.3 & 3.3 \\
\hline 7 & 116.7 & 143.3 & 133.3 & 133.3 & 13.3 \\
\hline 8 & 111.7 & 126.7 & 131.7 & 131.7 & 7.5 \\
\hline average & 117.3 & 130.8 & 130.2 & 129.4 & 7.2 \\
\hline
\end{tabular}




\section{Experiment 3}

The 7 subjects did not participate in previous experiments. The setting was exactly as in Experiment 2 with the only difference being that we doubled time for the first and the last tests of the day. This should counter-balance any practice, "warming-up" or different test difficulty effects in the previous experiment. The results are in Table 3 . The $\Delta$ values we computed as

$$
\Delta=\frac{S^{5}+S^{8}}{2}-\frac{S^{6}+S^{7}}{2}
$$

The average increase in effective IQ due to testing time increase is 5.4 points, the standard error is 2.2. The increase is noticeably less than in Experiment 2 and for one subject we even see a decrease.

Table 3. The clear columns show the IQ scores received by the subjects completing the test during standard time. The shaded columns show the scores received when the time allowance was increased twice. On average, the effective IQ measuured when the test time was increased twice is 5.4 points higher than the average IQ measured normally.

\begin{tabular}{|c|c|c|c|c|c|}
\hline $\begin{array}{c}\text { Kest \# } \\
\text { Subject \# }\end{array}$ & 5 & 6 & 7 & 8 & $\Delta$ \\
\hline 1 & 118.3 & 115.0 & 123.3 & 126.7 & 3.3 \\
\hline 2 & 123.3 & 120.0 & 125.0 & 130.0 & 4.2 \\
\hline 3 & 130.0 & 115.0 & 125.0 & 135.0 & 12.5 \\
\hline 4 & 126.7 & 118.3 & 120.0 & 118.3 & 3.3 \\
\hline 5 & 115.0 & 121.7 & 110.0 & 135.0 & 9.2 \\
\hline 6 & 115.0 & 120.0 & 126.7 & 121.7 & -5.0 \\
\hline 7 & 126.7 & 116.7 & 123.3 & 133.3 & 10.0 \\
\hline average & 122.1 & 118.1 & 121.9 & 128.6 & 5.4 \\
\hline
\end{tabular}

Analysis

We should cancel out all practice and different test difficulty effects if we average the results of the second and third experiment using the equation

$$
\bar{\Delta}=\frac{\overline{\Delta_{2}}+\overline{\Delta_{3}}}{2}
$$

where $\overline{\Delta_{2}}$ and $\overline{\Delta_{3}}$ are the average $\Delta$ values obtained in the second and third experiments. The result is 6.2 points with the standard error of 1.2 points.

We did not have the resources to do the counter-balance experiment for the Experiment 1 in the same manner as Experiment 3 counter-balances Experiment 2. So we did not use the data of Experiment 1 in our final estimate. We will just note that the results of the first experiment are perfectly consistent with it.

However, we cannot complete the analysis without clarifying some apparent anomalies in test results. In particular the 12-point increase in the average IQ between \#5 and \#8 tests in Experiment 2 and negative $\Delta$ for subject \#6 in Experiment 3. 
Practice effect and different difficulty levels of the different forms of IQ tests is nothing new. Thorndike (1922) observed those using his test prepared originally for use by the Examining Boards of the United States Air Service. He prepared fifteen alternative forms of the test. In a series of experiments, he gave couples of these forms to different groups of college entrants in different order. A ten minutes of fore-exercise using problems from other forms, not used in the test, preceded the tests. For example, when he first gave form $\mathrm{D}$ and then form $\mathrm{E}$, the average score difference $E-D$ was 4 points. If he first gave $\mathrm{E}$ and $\mathrm{D}$ afterward the average score difference $\mathrm{D}-\mathrm{E}$ was 12 points. By averaging these numbers Thorndike obtained that the score increase between the first and the second tests due to practice effect is 8 points. There is also a 4-point difference in difficulties between tests $\mathrm{E}$ and $\mathrm{D}$ which works in opposite direction with the practice effect in the first experiment and in the same direction in the second. Thorndike also measured the score differences due to practice effect between third and second tests, fourth and third and so on but they were much smaller than the difference between the second and the first test.

Taking these effects into account we can write the average scores obtained in Experiments 2 and 3 in a linear approximation in the following way:

$$
\begin{array}{ll}
\overline{S_{2}^{5}}=\overline{I Q_{2}}-D^{5}+P^{1} & \overline{S_{3}^{5}}=\overline{I Q_{3}}-D^{5}+P^{1}+T D \\
\overline{S_{2}^{6}}=\overline{I Q_{2}}-D^{6}+P^{2}+T D & \overline{S_{3}^{6}}=\overline{I Q_{3}}-D^{6}+P^{2} \\
\overline{S_{2}^{7}}=\overline{I Q_{2}}-D^{7}+P^{3}+T D & \overline{S_{3}^{7}}=\overline{I Q_{3}}-D^{7}+P^{3} \\
\overline{S_{2}^{8}}=\overline{I Q_{2}}-D^{8}+P^{4} & \overline{S_{3}^{8}}=\overline{I Q_{3}}-D^{8}+P^{4}+T D
\end{array}
$$

Here, $(i) \overline{S_{2}^{n}}$ and $\overline{S_{3}^{n}}$ are the average scores of our test subjects on $n^{\text {th }}$ test in experiments 2 and 3 respectively. Here $n$ refers to the test number in the book of Eysenck and Evans (1994). (ii) $D^{n}$ is the difficulty correction for the $n^{\text {th }}$ test. (iii) $P^{m}$ is the practice effect correction for $m^{\text {th }}$ test, where $m$ is the sequential test number in the experiment, $(i v) \overline{I Q_{2}}$ and $\overline{I Q_{3}}$ are the average IQs of the subjects of Experiments 2 and 3. Finally, (v) TD is the score gain due to time allowance doubling.

Using Equations (6) we get for the average $\Delta$ in Experiments 2 and 3:

$$
\begin{aligned}
& \overline{\Delta_{2}}=\frac{\overline{S_{2}^{6}}+\overline{S_{2}^{7}}}{2}-\frac{\overline{S_{2}^{5}}+\overline{S_{2}^{8}}}{2}=\mathrm{TD}-\frac{\mathrm{D}^{5}+\mathrm{D}^{8}-\mathrm{D}^{6}-\mathrm{D}^{7}}{2}+\frac{\mathrm{P}^{1}+\mathrm{P}^{4}-\mathrm{P}^{2}-\mathrm{P}^{3}}{2} \\
& \overline{\Delta_{3}}=\frac{\overline{S_{3}^{5}}+\overline{S_{3}^{8}}}{2}-\frac{\overline{S_{3}^{6}}+\overline{S_{3}^{7}}}{2}=T D+\frac{D^{5}+D^{8}-D^{6}-D^{7}}{2}-\frac{P^{1}+P^{4}-P^{2}-P^{3}}{2}
\end{aligned}
$$

Buy summing Equations (7) we get

$$
\overline{\Delta_{2}}+\overline{\Delta_{3}}=2 T D
$$

Which means that the intuitive Eq.(5) indeed gives us the effect of test time doubling, while canceling out the effect of all other influences in a first-order approximation.

Now let us turn to the negative $\Delta$ for subject \#6 in Experiment 3. If we look at the distribution of the individual score differences between tests 5 and 8 in Experiment 2 we see that it has mean 12.1 and the standard deviation (SD) 7.1. For the distribution of the individual score differences between tests 6 and 7 in Experiment 3 we get mean 3.8 and SD 7.3. This suggests that apart from the predictable 
score differences which are due to the inequality of test difficulty and practice effect there is also a random component.

Elo (1978) studied such randomness in the case of chess. In his model a player has certain average strength which can vary only slowly with time. But in every particular game his displayed strength consists of his average strength plus a random component. Thus, we cannot know for sure the outcome of a game between a stronger and a weaker player but can accurately predict its probability.

In IQ science they do control for random effects by studying test-retest reliability (Mackintosh, 1998). They measure this reliability as a correlation between test and retest scores. It is about 0.9 (Mackintosh, 1998). Let us try to estimate the standard deviation of test-retest score differences using this correlation value. Suppose that everyone has an intrinsic IQ and that the distribution of this intrinsic IQ among the people has SD $\tilde{\sigma}$. The measured IQ will be this intrinsic IQ plus some random variable $R$. We will denote the SD of this random variable as $\sigma_{R}$. If $R$ is uncorrelated with the intrinsic IQ the measured IQ will have the SD $\sigma=\sqrt{\sigma_{R}{ }^{2}+\tilde{\sigma}^{2}}$. A straightforward calculation gives for testretest correlation $r=\tilde{\sigma}^{2} / \sigma^{2}=1-{\sigma_{R}}^{2} / \sigma^{2}$. Therefore

$$
\sigma_{R}=\sigma \sqrt{1-r}
$$

By substituting $\sigma=15$ and $r=0.9$ into Eq.(9) we get $\sigma_{R}=4$.7. For the SD of the score differences between two tests we get $\sigma_{D}=\sqrt{2} \sigma_{R}=6.7$ which is close to what we got in our experiments. For the $\Delta$ we get: $\Delta_{2, \mathrm{i}}-\overline{\Delta_{2}}=\frac{\mathrm{R}_{1}+\mathrm{R}_{2}-\mathrm{R}_{3}-\mathrm{R}_{4}}{2}$. Here $\Delta_{2, \mathrm{i}}$ is the $\Delta$ for subject $\# i$ in Experiment $2, \mathrm{R}_{n}$ are independent random variables with mean 0 and SD of $\sigma_{R}$. We can simplify the equation to $\Delta_{2, \mathrm{i}}-$ $\overline{\Delta_{2}}=R$. A similar equation holds for Experiment 3. When we combine $\Delta_{2, \mathrm{i}}-\overline{\Delta_{2}}$ and $\Delta_{3, \mathrm{i}}-\overline{\Delta_{3}}$ we get an SD of 4.4, very close to the 4.7 value we obtained from test-retest reliability studies. The anomalous data point with negative $\Delta$ deviates a little more than 2 SD. The probability to get one data point out of 15 deviate more than $2 S D$ is $28 \%$. This is well within the limits of the law of chances.

We conclude that there are really no anomalies in our data and that our estimate of the effect of test time doubling on measured IQ is sound.

\section{Discussion}

This approach to interpreting IQ seems rather obvious and we expected to find it in a textbook. To no avail. Few things had been tangentially relevant.

Cronbach (1949) in Table 23 of his book lists the results of the unpublished Doctor's thesis of K.C. Eells. Eells approximately doubled the prescribed test times for Otis and Henmon-Nelson tests and reported mean points earned in additional time. We cannot be sure what was the aim of Eells, but Cronbach uses his results just to discuss what should be the right time limit for the tests. Jensen (1980) discusses these results in his book but only says that the time limit is not important if it does not change the rank-order of the tested.

Eysenck (1953) described some interesting experiments of Furneaux (his own account is also available (Furneaux , 1960) but is much less readable). Furneaux measured the difficulty of a problem as 1 minus the fraction of the test subjects who solved it correctly given no time limit. He plotted the time taken to solve problems versus their difficulty. He observed that these curves became straight lines when he used a logarithmic scale for time. He also observed that these lines for subjects of different 
intelligence were parallel to each other. This means that while people of different intelligence take different time to solve an easy problem $\mathrm{A}$ or a difficult problem $\mathrm{B}$, the ratio of the time to solve $\mathrm{B}$ to the time to solve $\mathrm{A}$ is approximately the same for all people. One can define the speed of thinking of a particular person as an inverse of time required to solve a reference problem. This is like the Intellectual Power concept we will introduce later. In discussing Furneaux experiments Eysenck says:

It must be remembered, of course, that our time scale is a logarithmic scale, so that the required increase in time to solve a difficult problem is quite disproportionate for the dull person; he might require several months of persistent application to solve a problem which the bright person could solve in a few minutes.

However, Eysenck did not go anywhere further than that. He did not propose any quantitative relation between the time increase and IQ difference ${ }^{3}$.

In an interview (Warne, 2018a) intelligence researcher Russel Warne have said: "you can compensate for 5-10-15 who knows may be more points deficit by working hard." However, when contacted to clarify if there is experimental data supporting these figures he responded with a clever heuristic argument:

I found an important article on the subject (Schmidt \& Hunter, 2004, Table 3), which lists the correlation between IQ and performance on the job for ten occupations. The average and median correlation are both almost exactly $\mathrm{r}=.55$. Assuming that this correlation is typical, then it indicates that two people who are 5 points (.33 SD's) apart on IQ will be--on average--.183 SD's apart on job performance, with the smarter person being better at the job. Two people who are 10 points (.66 SD's) apart on IQ will be--on average--.367 SD's apart on job performance, with (again) the smarter person being better at the job. (Both estimates found by multiplying the SD difference in IQ by the $\mathrm{r}$ value of .55.)

In the real world, differences of .20 SD's are about the minimum differences that can be noticed in daily life by a carefully observant layman. So, a boss probably wouldn't notice the differences in job performance for two employees who are 5 IQ points apart but equal on all other variables. A lower-IQ employee could probably easily compensate the difference with a stronger work ethic.

For the 10-point gap, Even the differences between employees with a 10-point IQ gap are not so large (on average) that a stronger work ethic, extra enthusiasm, and other variables can compensate for the lower scoring employee's IQ disadvantage.

The results of our direct experiment suggest that by working twice longer one can compensate for 6 IQ points. It is probably impossible to compensate for more unless the higher IQ competitor is lazy.

\footnotetext{
${ }^{3}$ We also would like to note that part of Furneaux results appear counter-intuitive. Namely his definition of difficulty suggest that the difference in it between the problem unsolved by $99 \%$ of the tested and that unsolved by $90 \%$ is the same as the difficulty difference between the problems unsolved by $59 \%$ and $50 \%$. And leads to the same relative increase in time required to solve the problem by those who manage to solve it. If we set to determine the probability density distribution of the speed of thinking, $s$, among the test subjects which could lead to these results we get $p(s) \sim 1 / s$. This is impossible at the high- $s$ end. Probably Furneaux experiment did not include really difficult problems.
} 
Since Warne had to resort to a heuristic argument, we suspect that our approach will be new for the intelligence researchers. At least it is not widely known. It is likely, however, that there is an old unknown paper pushing similar ideas for the scientists re-invent things very often (Simkin and Roychowdhury, 2011).

Dunham (2014) in a lecture about Isaac Newton have said: "He has an IQ that's three digits, four digits, five digits, who knows." If we are talking about the traditionally defined IQ, then we do know that it was three digits. For to have an IQ of 1000 Newton had to have a normal 16-year-old intelligence at the age of a year and a half. We can get the same result using Normal distribution. A 200 IQ may be feasible. It is $100 / 15 \cong 6.66$ standard deviations above the average and the frequency of such an IQ or bigger is $\frac{1}{2}(1-\operatorname{erf}(6.66 / \sqrt{2})) \cong 1.3 \times 10^{-11}$. With $7 \times 10^{9}$ world population the probability that someone alive today has such an IQ is $9 \times 10^{-2}$. However, the probability to have an IQ of 1000 is $1.2 \times 10^{-784}$ which is essentially 0 . So, Newton's IQ was three digits indeed.

But may be Dunham intuitively equated IQ with the speed of problem solving? In this case a five digits IQ (that is $10^{4}$ or more) means that the person with this IQ can solve problems 100 times faster than an average person who has an IQ of 100. Our experiment implies that problem solving speed increases twice with an increase of IQ by 6 points. Suppose that this relation goes forever. That is with each increase of IQ by 6 points the problem-solving speed increases twice. This does not necessarily follow from our experiment but does not contradict it as well. Let us call the problemsolving speed "Intellectual Power" and denote it as IP. We then have $I P(I Q)=100 \times 2^{(I Q-100) / 6}$. Here we define $I P(100)=100$, that is for an average man IP equals IQ. By reversing this equation, we get $I Q(I P)=6 \times \log _{2}(I P / 100)+100$. From this equation we get $I Q(10,000) \cong 140$. So a 5 digit IP is perfectly possible. Note, however that at present this is just a guess. To check whether it has anything to do with reality one would have to do some more experiments increasing test time allowance 4 and 8 times.

\section{References}

Cronbach, L. J. (1949). Essentials of Psychological Testing. New York: Harper, 1949.

Dunham, W. (2014). Your humble Servant, Is. Newton. MAA Distinguished Lecture: https://www.maa.org/meetings/calendar-events/your-humble-servant-is-newton , https://www.youtube.com/watch?v=tQ5D8zRr2IA

Elo, A.E. (1978). The rating of chess players, past and present. New York: Arco publishing. Eysenck, H. (1953). Uses and Abuses of Psychology. Harmondsworth: Penguin.

Eysenck, H., Fulker, D. W. (1979). The Structure and Measurement of Intelligence. Berlin: Springer.

Eysenck, H., Evans, D. (1994). Test your IQ. New York: Penguin.

Furneaux, W.D. (1960). Intellectual Abilities and Problem-solving Behaviour. In H. J. Eysenck (Ed.), Handbook of abnormal psychology (pp. 167-192). London: Pitman Medical

Gould, S. J. (1996). The Mismeasure of Man. New York: Norton. 
Herrnstein, R.J. and Murray, C. (1994). The Bell Curve: Intelligence and Class Structure in American Life. New York: The Free Press.

Jensen, A. R. (1980). Bias in mental testing. New York: Free Press.

Jensen, A. R. (1998). The g factor: The science of mental ability. Westport, CT: Praeger.

Mackintosh, N.J. (1998). IQ and Human Intelligence. Oxford University Press.

Schmidt, F. L., Hunter, J. (2004). General Mental Ability in the World of Work: Occupational Attainment and Job Performance. Journal of Personality and Social Psychology, Vol 86(1), 162-173.

Simkin, M.V. and Roychowdhury, V.P. (2011) Re-inventing Willis, Physics Reports, 502, 1-35.

Thorndike, E.L. (1922). Practice effects in intelligence tests. Journal of Experimental Psychology, 5, 101-107.

Warne, R. (2018a). Wrong About IQ? I Russell Warne and Stefan Molyneux.

https://www.youtube.com/watch?v=EQO8I-j3Eac

Warne, R. (2018b). Private communication. 